\title{
Применение статистических методов для учета влияния рельефа местности при проведении аэромагнитных съемок
}

\author{
(C)2020 О. М. Муравина ${ }^{1 凶}$, А. С. Долгаль ${ }^{2}$, И. А. Пономаренко ${ }^{1}$ \\ ${ }^{1}$ Воронежский государственный университет, \\ Университетская пл., 1, 394018, Воронеж, Российская Федерация \\ ${ }^{2}$ Горный институт УрО РАН, \\ ул. Сибирская, 78а, 614007, Нермь, Российская Федерация
}

\begin{abstract}
Аннотация
Bведение: В работе представлена методика применения нового статистического подхода для учета влияния дневного рельефа, сложенного намагниченными магматическими породами, на результаты измерений магнитного поля.

Методика: Предложена компьютерная технология, основанная на синтезе метода эмпирической модовой декомпозиции (EMD) и метода группового учета аргументов (МГУА). Эта технология реализована при обработке данных аэромагниторазведки, выполненной в сложных физико-геологических условиях западной части плато Путорана.

Результаты и обсуждение: Выполнено разложение дискретных значений аномального магнитного поля $\Delta \mathrm{T}$ и высотных отметок рельефа Н на эмпирические модовые функции (IMF); проведено идентификационное моделирование МГУА для десяти опытных профилей, по результатам которого оптимизирована методика дальнейшего анализа. Для каждого профиля для определенных составляющих магнитного поля получены уникальные уравнения их взаимосвязи с рельефом, рассчитаны модельные значения составляющих и выполнена оценка расхождения первичных и модельных значений компонент магнитного поля.

Заключение: Результаты выполненных исследований позволили отработать практические приемы реализации предлагаемого авторами нового подхода учета влияния рельефа местности на данные аэромагниторазведки. Обобщенные характеристики моделей, полученные для всего объема данных, согласуются с результатами на опытных профилях, что подтверждает правильность предварительных оценок и правомерность предложенной последовательности выполнения анализа. Выявленные взаимосвязи между компонентами магнитного поля и рельефа позволили осуцествить подавление влияния траппового покрова на аномальное магнитное поле и обеспечили возможность выделения слабо проявленных геологических тел и структур.

Ключевые слова: метод эмпирической модовой декомпозиции, метод группового учета аргументов, аэромагниторазведка, учет влияния рельефа.

Источник финансирования: работа выполнена при поддержке грантов РФФИ № 19-05-00654 А, 18 05-70094 «Ресурсы Арктики».

Для циитирования: Муравина О. М., Долгаль А. С., Пономаренко И.А. Применение статистических методов для учета влияния рельефа местности при проведении аэромагнитных съемок // Becmник Воронежского государственного университета. Серия: Геология. 2020. №2. С. 69-75. DOI: https://doi.org/10.17308/geology.2020.2/2860
\end{abstract}

Контент доступен под лицензией Creative Commons Attribution 4.0 License.

\footnotetext{
๑ Муравина Ольга Михайловна, e-mail: muravina@geol.vsu.ru
} 


\section{Введение}

Учет влияния рельефа местности на результаты магнитной съемки (в частности аэромагниторазведки) является важной и трудно реализуемой задачей в условиях значительного перепада высот и неоднородности магнитных свойств пород верхней части разреза в пределах больших магматических провинций [1]. Существуют различные подходы к ее решению, как детерминированные [2, 3], так и стохастические [4], весьма редко применяющиеся на практике. Аномальные эффекты, обусловленные рельефом местности, при решении практических задач, как правило, выявляются на стадии качественной интерпретации карт аномального магнитного поля, при совмещении последних с топографическими картами. Это позволяет осуществлять визуальную разбраковку магнитных аномалий на два типа: «топографические» и «геологические» (условно), однако не исключает возможности пропуска искомых магнитовозмущающих объектов (ошибок I рода) и выделения ложных аномалий, отождествляемых с искомыми геологическими объектами (ошибок II рода) [5].

Необходимость подготовки комплектов геологических карт нового поколения, а также поисков месторождений полезных ископаемых в областях развития платобазальтов требуют разработки новых инновационных способов ее решения [3]. Следует отметить, что возможности детерминированных методов, основанных на решении прямой задачи магниторазведки, существенно ограничивает дефицит петрофизической информации и отсутствие данных о высоте съемочных полетов практически для всех аэромагнитных съемок, выполненных в $\mathrm{XX}$ веке на территории России. Построение достоверных карт эффективной намагниченности горных пород $J_{э ф}$ верхней части геологического разреза, отвечающих масштабу исследований, не представляется возможным: затраты на получение необходимого объема петрофизической информации многократно превышают затраты на выполнение самой съемки [6].

Типичной ситуацией является выполнение крупномасштабных аэромагниторазведочных работ на площади в несколько тысяч кв. км, охарактеризованной петрофизическими измерениями первых десятков образцов (как правило - не ориентированных, что не позволяет определять вектор остаточной намагниченности). Весьма грубым приближением является также использование некоторой, средней для всей площади ис-

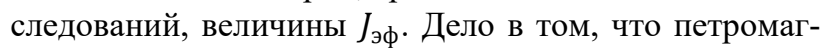
нитные параметры магматических горных пород обладают высокой латеральной изменчивостью; в современном эрозионном срезе эти породы могут контактировать с практически немагнитными осадочными отложениями.

Поэтому при описании магнитных свойств рельефа в первом приближении суммарная намагниченность пород является функцией от горизонтальных координат: $J_{э \phi}=f(x, y)$. При этом мы вынуждены пренебрегать вертикальным изменением магнитных свойств $J_{\text {эф }}=\varphi(z)$, так как в реальности наблюдается субгоризонтальное залегание горных пород в виде покровов эффузивов и интрузивных (субвулканических) залежей, переслаивающихся с континентальными и лагунными осадками, иногда с туфами [4]. Установлено, что над платобазальтами статистическая взаимосвязь амплитуд магнитных аномалий и высотных отметок рельефа носит весьма сложный характер, который не может быть описан с помощью линейной регрессии (рис. 1). В данном примере коэффициент детерминации равен 0.018 .

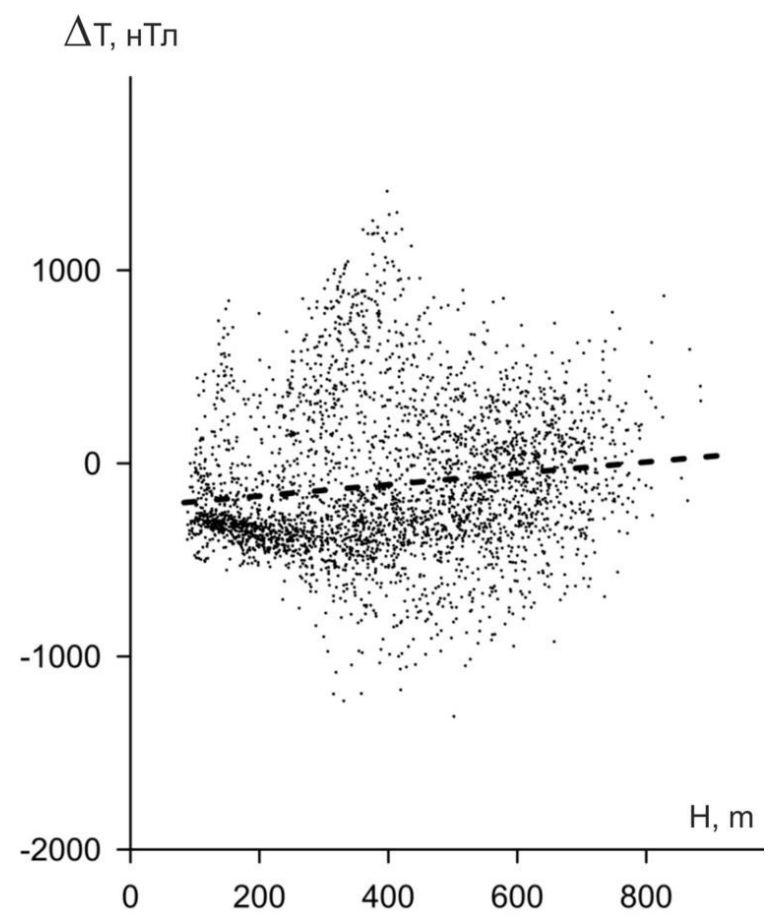

Рис. 1. Кросс-плот, характеризующий взаимосвязь между амлитудой магнитных аномалий $\Delta \mathrm{T}$ и высотами рельефа Н. Хараелахская трапповая мульда (Норильский район). Штрих-пунктир - линия регрессии: $\Delta \mathrm{T}=0.293 \mathrm{H}-227.5$ (объем выборки 12876 точек).

[Fig. 1. Cross-raft, characterizing the relationship between the amplitude of the magnetic anomalies $\Delta \mathrm{T}$ and the elevation of the relief $\mathrm{H}$. The Haraelakh trap trough (Norilsk region). The dash-dotted line is the regression line: $\Delta \mathrm{T}=0.293 \mathrm{H}-227.5$ (sample size -12876 points). ]

В 2017 г авторами был предложен принципиально новый стохастический способ определения поправки за рельеф, основанный на идентификации некоторых составляющих аномального поля в определенных диапазонах пространственных частот, которая наиболее тесно взаимосвязана (возможно - нелинейно) с высотами рельефа дневной поверхности [7]. Способ реализуется на основе синтеза метода эмпирической модовой декомпозиции (EMD) и метода группового учета аргументов (МГУА).

EMD позволяет в ходе итерационного вычислительного процесса адаптивно выделять ортогональные разночастотные компоненты сигнала, которые называются эмпирическими модовыми функциями (IMF). 
Метод предназначен для частотного представления нестационарных сигналов, его отличительной особенностью является отсутствие априорно заданного функционального базиса разложения [8, 9]. Метод группового учета аргументов выявляет неявные взаимосвязи между элементами сложных систем. В отличие от традиционного регрессионного анализа структура и параметры модельного уравнения не задаются заранее, а выбираются из множества сгенерированных вариантов по минимальному значению внешнего критерия [1011]. Исследования, выполненные на кафедре геофизики Воронежского госуниверситета, подтвердили эффективность использования МГУА при работе с геолого-геофизической информацией [11-18]. Первые результаты применения предложенного нового способа учета магнитного рельефа продемонстрировали его перспективность [7, 18, 19]. В настоящей работе приводятся методика и результаты его применения при интерпретации значительного объема данных аэромагниторазведки, выполненной в сложных физико-геологических условиях западной части плато Путорана (арктическая часть Восточной Сибири), на листе Q-46.

\section{Фактические материалы}

В качестве фактических материалов были использованы актуализированные результаты аэромагнитных съемок масштаба 1:100 000-1:50 000, выполненных в 1962-81 гг. и высотные отметки дневного рельефа, интерполированные в сеть $1 \times 1$ км. Общее число точек поля по 385 профилям составило 112805, площадь исследований - 112805 км² $^{2}$ соответственно. Данные о высотах съемочных полетов отсутствуют. Следует добавить, что подавляющее большинство материалов не удовлетворяет современным требованиям как по точности измерений ( 10-20 нТл), так и по качеству плановой привязки.

\section{Методика}

На первом этапе дискретные значения аномального магнитного поля $\Delta \mathrm{T}$ и высотные отметки рельефа $\mathrm{H}$ были разложены на эмпирические модовые функции (IMF) [9]. В результате для аномального магнитного поля по каждому профилю были получены семейства IMF ( $\delta T 1-\delta T 6)$, суммарное поле ( $\delta T$ sum) $u$ остаточная составляющая ( $\delta$ Tres). Высотные отметки рельефа были разложены на компоненты $(H 1-H 6)$, суммарную составляющую (Hsum) и остаточную составляющую (Hres). Результаты EMD затем были использованы в качестве исходных данных в процедуре идентификационного моделирования МГУА.

Для оптимизации процедуры моделирования было выбрано 10 профилей в наиболее сложной (по характеру поля и рельефа) части площади, для которых был выполнен полный комплекс идентификационного моделирования для всего набора частотных составляющих магнитного поля и высот рельефа. Полученные уравнения были проанализированы и на основе выполненного анализа определены наиболее информатив- ные с точки зрения решаемой задачи компоненты магнитного поля, а также параметры идентификационного моделирования.

В таблице 1 представлены минимаксные и средние значения критерия выбора оптимальной модели.

Данные табл. 1 указывают на то, что лучшим образом с рельефом коррелируются низкочастотные компоненты, которым соответствуют - IMF с большими номерами ( $\delta T 4-\delta T 6)$, суммарное поле ( $\delta T s u m) u$ остаточная составляющая ( $\delta$ Tres).

Табл. 1. Диапазоны изменения внешнего критерия для опытных профилей

[Table 1. Ranges of change of external criterion for experienced profiles]

\begin{tabular}{|c|c|c|c|}
\hline $\begin{array}{c}\text { Компонента } \\
\text { [Component] }\end{array}$ & $\varepsilon_{\text {мин }}$ & $\varepsilon_{\text {макс }}$ & $\varepsilon_{\text {сред }}$ \\
\hline$\delta T 1$ & 0.40 & 0.93 & 0.54 \\
\hline$\delta T 2$ & 0.43 & 0.92 & 0.67 \\
\hline$\delta T 3$ & 0.50 & 0.80 & 0.64 \\
\hline$\delta T 4$ & 0.30 & 0.76 & 0.51 \\
\hline$\delta T 5$ & 0.24 & 0.65 & 0.46 \\
\hline$\delta T 6$ & 0.28 & 0.78 & 0.50 \\
\hline$\delta T$ sum & 0.20 & 0.47 & 0.28 \\
\hline$\delta$ Tres & 0.07 & 0.62 & 0.17 \\
\hline
\end{tabular}

Приведем характеристики моделей вышеназванных компонент магнитного поля, полученные на опытных профилях.

Bce оптимальные модели (46 штук) получены на первом ряду селекционного отбора [13], поэтому имеют единую структуру, представляющую зависимость вида:

$$
\delta T_{k}=a_{0}+a_{1} H_{i}+a_{2} H_{j}+a_{3} H_{i} \cdot H_{j},
$$

где $\delta T_{k}-\mathrm{IMF}$-компонента магнитного поля, выступающая в качестве зависимой переменной, отождествляемая с поправкой за влияние магнитного рельефа; $H_{i}$, $H_{j}$ - IMF-компоненты высотных отметок; $a_{0}, a_{1}, a_{2}, a_{3}-$ числовые коэффициенты.

Представление о том, с какими компонентами рельефа чаще коррелируется составляющие магнитного поля, дает диаграмма, представленная на рис. 2.

Как очевидно, наиболее часто в модельных уравнениях встречаются компоненты H5 (30 моделей), Hres (27 моделей), Hsum (27 моделей).

В моделях, в которых зависимой переменной является одна из компонент $\delta T 4$ - $\delta T 6$, также наиболее часто встречается компонента H5, Hres и Hsum, а также присутствует компонента $H 4$. В оптимальных уравнениях для $\delta T$ sum и $\delta$ Tres одной из переменных-аргументов является аналогичная компонента рельефа Hres и Hsum, соответственно.

Самыми распространенными стали 4 вида моделей:

1) $1-\delta T k=a 0+a 1 H 5+a 2 H r e s+a 3 H 5 \cdot H r e s-12$ моделей, что соответствует $26 \%$ от общего числа; 
2) $2-\delta T k=a 0+a 1 H 5+a 2 H S u m+a 3 H 5 \cdot H S u m-12$ моделей, что соответствует $26 \%$ от общего числа;

3) $3-\delta T k=a 0+a 1 H r e s+a 2 H S u m+a 3 H r e s \cdot H S u m-7$ моделей, что соответствует $15 \%$ от общего числа;

4) 4 - $\delta T k=a 0+a 1 H 4+a 2 H r e s+a 3 H 4 \cdot H r e s-6$ моделей, что соответствует $13 \%$ от общего числа модельных уравнений;

На остальные 5 вариантов модельных уравнений приходится $13 \%$ от общего числа.

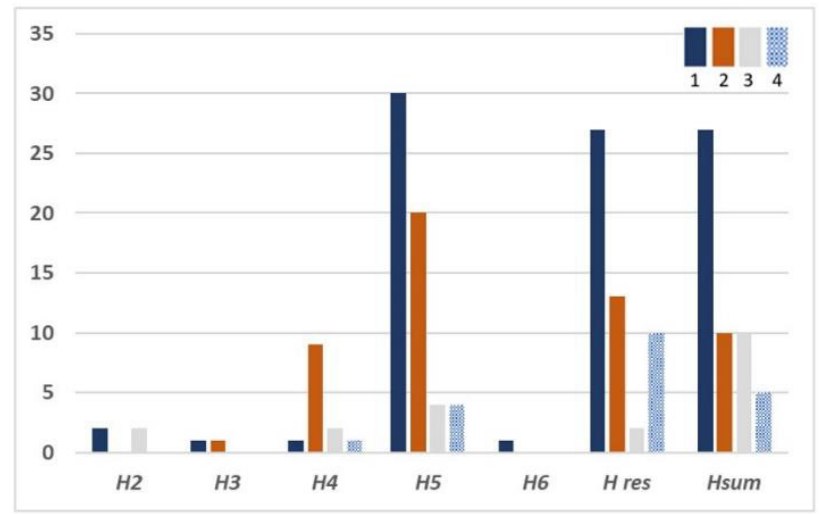

Рис. 2. Частота встречаемости компонент рельефа (H2-H6, Hres, HSum) в моделях: 1 - все модели; 2 - модели $\delta T 4$ - $\delta T 6$;

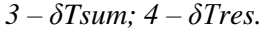

[Fig. 2. Frequency of occurrence of relief components $(\mathrm{H} 2-\mathrm{H} 6$, Hres, HSum) in the models: 1 -all models; 2 - models $\delta T 4$ -

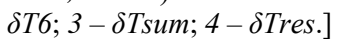

На следующем этапе идентификационное моделирования было выполнено для всего объема данных. Модельные уравнения рассчитывались только для отобранных на предыдущем этапе магнитных составляющих: $\delta \mathrm{T} 4-\delta \mathrm{T} 6$, суммарного поля $\delta \mathrm{Tsum} \mathrm{и} \mathrm{остаточного}$ поля $\delta$ Tres. Поиск оптимальных моделей осуществлялся только среди моделей первого ряда.

\section{Результаты}

Таким образом, выполнено разложение дискретных значений аномального магнитного поля $\Delta \mathrm{T}$ и высотных отметок рельефа Н на эмпирические модовые функции (IMF). Для десяти опытных профилей проведено идентификационное моделирование МГУА, по результатам которого определена оптимальная методика дальнейшего анализа. В результате для каждого профиля были получены модельные уравнения взаимосвязи составляющих магнитного поля и рельефа, рассчитаны модельные значения составляющих и выполнена оценка расхождения первичных и модельных значений компонент магнитного поля. На рис. 3, 4 приведены некоторые результаты идентификационного моделирования низкочастотных составляющих магнитного поля по профилю 11, позволяющие оценить соответствие экспериментальных и модельных значений.

Результаты анализа точности и параметры полученных моделей представлены в таблицах 2, 3. Обобщенные характеристики моделей, полученных для всего объема данных, согласуются с результатами, полученными на опытных профилях, что подтверждает правильность предварительных оценок.
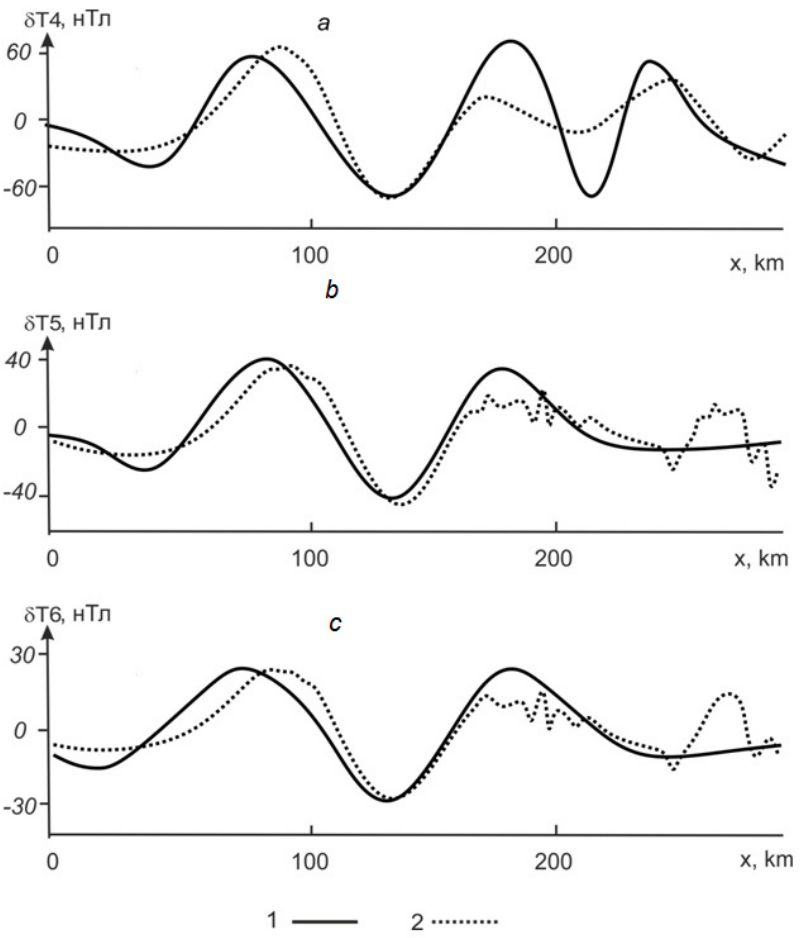

Рис. 3. Результаты идентификационного моделирования низкочастотных составляющих магнитного поля по профилю $11: a$-для составляющей $\delta \mathrm{T} 4 ; b$ - для составляющей $\delta \mathrm{T} 5 ; c$ - для составляющей $\delta \mathrm{T} 6: 1$ - экспериментальные, 2 - модельные значения.

[Fig. 3. Results of identification modeling of low-frequency components of the magnetic field along profile 11: $a$ - for component $\delta \mathrm{T} 4 ; b$ - for component $\delta \mathrm{T} 5 ; c$ - for component $\delta \mathrm{T} 6: 1$ - experimental, 2 - model values.]

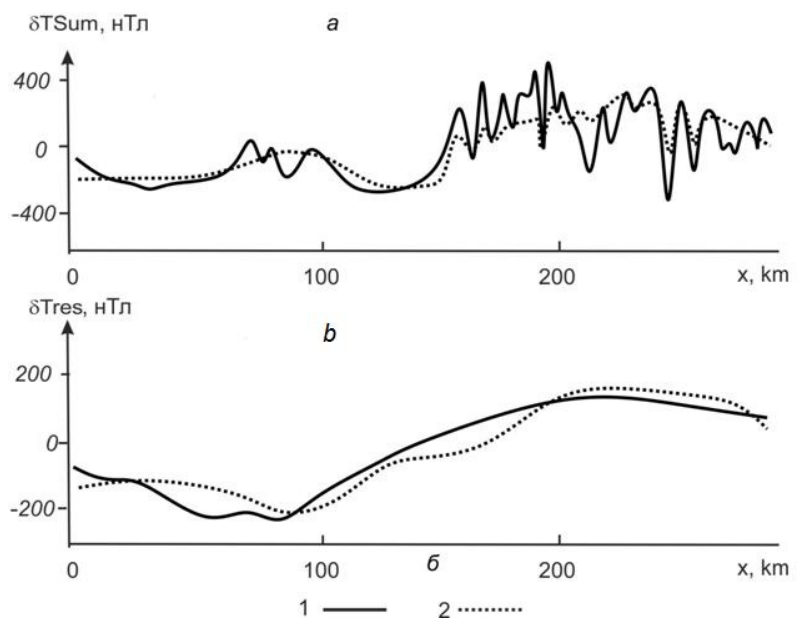

Рис. 4. Результаты идентификационного моделирования суммарной $\delta T$ sum (a) и остаточной $\delta$ Tres (b) составляющих магнитного поля по профилю 11: 1 - экспериментальные, 2 модельные значения.

[Fig. 4. The results of identification modeling of the total $\delta$ Tsum (a) and residual $\delta$ Tres (b) of the magnetic field components along the 11: 1 - profile experimental, 2 - model values.] 
Табл. 2. Диапазоны изменения внешнего критерия оптимальных моделей

[Table 2. Ranges of change of the external criterion of optimal models]

\begin{tabular}{|c|c|c|c|}
\hline $\begin{array}{c}\text { Компонента } \\
\text { [Component] }\end{array}$ & $\varepsilon_{\text {мин }}$ & $\varepsilon_{\text {макс }}$ & $\varepsilon_{\text {сред }}$ \\
\hline$\delta T 4$ & 0.13 & 0.97 & 0.66 \\
\hline$\delta T 5$ & 0.13 & 0.98 & 0.63 \\
\hline$\delta T 6$ & 0.16 & 0.97 & 0.67 \\
\hline$\delta$ Tsum & 0.11 & 0.89 & 0.48 \\
\hline$\delta$ Tres & 0.01 & 0.89 & 0.28 \\
\hline
\end{tabular}

Табл. 3. Параметры наиболее распространенных моделей

[Table 3. Parameters of the most common models]

\begin{tabular}{|c|c|c|c|c|c|}
\hline \multirow{2}{*}{$\begin{array}{c}\text { Переменные- } \\
\text { аргументы } \\
\text { [Argument } \\
\text { Variables] }\end{array}$} & \multicolumn{5}{|c|}{$\begin{array}{c}\text { Зависимые переменные } \\
\text { [Dependent Variables] }\end{array}$} \\
\hline & $\delta T 4$ & $\delta T 5$ & $\delta T 6$ & sTres & STSum \\
\hline$H 4, H 5$ & $15 \%$ & $10 \%$ & $17 \%$ & $0,3 \%$ & $0,3 \%$ \\
\hline H4,Hres & $21 \%$ & $20 \%$ & $17 \%$ & $7 \%$ & $5 \%$ \\
\hline H5,Hres & $21 \%$ & $23 \%$ & $17 \%$ & $18 \%$ & $12 \%$ \\
\hline Hres, HSum & $11 \%$ & $16 \%$ & $20 \%$ & $47 \%$ & $56 \%$ \\
\hline
\end{tabular}

\section{Заключение}

Результаты выполненных исследований позволили отработать практические приемы реализации предлагаемого авторами нового подхода учета влияния рельефа местности на данные аэромагниторазведки.

Обобщенные характеристики моделей, полученные для всего объема данных, согласуются с результатами, полученными на опытных профилях, что подтверждает правильность предварительных оценок.

Выявленные взаимосвязи между компонентами магнитного поля и рельефа позволили осуществить подавление влияния рельефа местности на аномальное магнитное поле и обеспечили возможность выделения слабо проявленных геологических тел и структур.

Благодарности: Авторы выражают благодарность проф. В. Н. Глазневу за конструктивные замечания и полезное обсуждение материалов исследования.

Конфликт интересов: Авторы декларируют отсутствие явных и потенциальных конфликтов интересов, связанных с публикацией настоящей статьи.

\section{ЛИТЕРАТУРА}

1. Криволуцкая Н. А. Эволюция траппового магматизма и $\mathrm{Pt}$ $\mathrm{Cu}-\mathrm{Ni}$ рудообразования в Норильском районе. Товарищество научных изданий КМК. 2014. 305 с.

2. Ремпель Г. Г. Актуальные вопросы методики введения поправок, связанных с рельефом местности данные гравиразведки и магниторазведки // Изв. АН СССР.Сер. Физика Земли. 1980. № 12. C. $75-89$.

3. Долгаль А. С., Лазарев Ф. Д., Канунников В. А., Кирплюк П. В. Учет влияния рельефа местности на результаты крупномасштабной аэромагнитной съемки. Вопросы теории и практики геологической интерпретации гравитационных, магнитных и электрических полей: материалы 42-й сессии Междунар. науч. семинара им. Д.Г. Успенского. Пермь, 26-30 января 2015. Горный институт УрО РАН, Перм. нац. Исслед. ун-т. Пермь, 2015. C. 73-75.
4. Долгаль А. С. Магниторазведка: компьютерные технологии учета влияния рельефа местности. Пермь, Изд-во Перм. гос. нац. исслед. ун-та. 2014. 92 с.

5. Dolgal A. S. Allowance for the Earth's Surface Topography in Processing the Magnetic Field Measurements. In: Nurgaliev D., Khairullina N. (eds) Practical and Theoretical Aspects of Geological Interpretation of Gravitational, Magnetic and Electric Fields. Springer Proceedings in Earth and Environmental Sciences. Springer, Cham. 2019. pp 3-12. DOI https://doi.org/10.1007/978-3-31997670-9 12 .

6. Долгаль А. С., Червоный Н. П. Учет влияния рельефа земной поверхности при аэромагнитных измерениях. // Геоінформатика. 2008. № 2. С. 58-66.

7. Dolgal A. S., Muravina O. M., Hristenko L. A. The reduction of the magnetic field within development areas of the plateaubasalts // Geoinformatics 2017. 15-17 May 2017, Kiev, Ukraine. 11143_ENG. Электронная публикация (CD).

8. Huang N. E., Shen Z.,. Long S. R, Wu M. C., Shih H. Y., Zheng Q., Yen N. C., Tung C. C., and Liu H. H. The empirical mode decomposition and the Hilbert spectrum for nonlinear and non-stationary time series analysis // Proc. R. Soc. Lond. A. 1998. Vol. 454, pp. 903-995. 9. Долгаль А. С., Христенко Л. А. Применение эмпирической модовой декомпозиции при обработке геофизических данных // Известия Томского политехнического университета. Инжиниринг георесурсов. 2017. Т. 328. № 1. С. 100-108.

10. Ивахненко А. Г. Индуктивный метод самоорганизации моделей сложных систем. Киев: Наукова думка. 1982. 296 с

11. Муравина О. М. Метод группового учета аргументов при анализе геофизических данных // Геофизика. 2012. № 6. С. 10-20.

12. Muravina O.M. Davudova I. A. Ponomarenko I. A. Possibility of identification of modeling in complex analysis geological and geophysical data. Practical and Theoretical Aspects of Geological Interpretation of Gravitational, Magnetic and Electric Fields: proceedings of the 45th Uspensky International Geophysical Seminar. Cep. "Springer Proceedings in Earth and Environmental Sciences". 2019, pp. 157-162.

13. Муравина О. М., Пономаренко И. А. Программная реализация метода группового учета аргументов при идентификационном моделировании геолого-геофизических данных // // Вестник Воронежского государственного университета. Серия: Геология. 2016. №2. С. 107-110.

14. Муравина О. М., Аузин А. А., Глазнев В. Н., Пономаренко И. А. О возможности изучения коллекторских свойств пород методом идентификационного моделирования // Каротажник. 2018. № 8. C.71-80.

15. Муравина О. М. Идентификационный анализ петрофизических характе-ристик пород осадочного чехла Воронежской антеклизы // Вестник КРАУНЦ. Сер. Науки о Земле. 2013. Т. 22. № 2. C. $20-25$.

16. Муравина О. М. Возможности метода группового учета аргументов при статистической обработке петрофизических данных // Вестник Воронежского государственного университета. Сер. Геология. 2009. № 2. С. 150-110.

17. Муравина О. М., Чернышова М. Н., Жаворонкин В. И. Идентификационный анализ ультрамафит-мафитовых интрузий мамонского комплекса Воронежского кристаллического массива // Вестник КРАУНЦ. Сер. Науки о Земле. 2019. Т. 43. № 3. С.8998. DOI: $\quad 10.31431 / 1816-5524-019-3-43-89-98$

18. Муравина О. М., Долгаль А. С., Аузин А. А., Пономаренко И. А., Груздев В.Н. Сферы применения современных статистических методов обработки геофизической информации // // Вестник Воронежского государственного университета. Серия: Геология. 2019. № 4. С. 79-84.

19. Долгаль А. С., Муравина О. М., Криволуцкая Н. А. Учет влияния рельефа земной поверхности при редуцировании аэромагнитных измерений в пределах больших магматических провинций. Глубинное строение, геодинамика, тепловое поле Земли: материалы десятых научных чтений памяти Ю.П. Булашевича, Екатеринбург, 2019. С. 114-118. 
UDC 550.831

DOI: https://doi.org/10.17308/geology.2020.2/2860

ISSN 1609-0691

Received: 11.05 .2020

Accepted: 01.06.2020

Published online: 30.06 .2020

\title{
Application of statistical methods to take into account the influence of the terrain when conducting aeromagnetic surveys
}

\author{
(C)2020 O. M. Muravina ${ }^{1 凶}$, A. S. Dolgal ${ }^{2}$, I. A. Ponomarenko ${ }^{1}$ \\ ${ }^{1}$ Voronezh State University, \\ 1 Universitetskaya pl., Voronezh 394018, Russian Federation \\ ${ }^{2}$ Mining Institute of the Ural Branch of the Russian Academy of Sciences, \\ 78 a Sibirskaya st., Perm 614007, Russian Federation
}

\begin{abstract}
Introduction: The study presents a method for applying a new statistical approach to take into account the effect of the daylight terrain, composed of magnetised magmatic rocks, on the results of magnetic field measurements. Methodology: A computer-based technology is proposed that is based on the synthesis of the method of empirical mode decomposition (EMD) and the group method of data handling (GMDH). This technology is implemented in the processing of the data from aeromagnetic surveys, performed in difficult physical and geological conditions of the western part of the Putorana plateau.

Results and discussion: The discrete values of the anomalous magnetic field $\Delta \mathrm{T}$ and the relief elevations $\mathrm{H}$ were decomposed into empirical mode functions (IMF). The GMDH identification modelling was conducted for ten experimental profiles. Based on this data, the methodology for the further analysis was optimised. For each profile, for certain components of the magnetic field, unique equations of their correlation with the terrain were obtained, model values of the components were calculated, and the discrepancy between the primary and model values of the components of the magnetic field was estimated.

Conclusions: The research results made it possible to apply the practices for implementing the new approach proposed by the authors to take into account the influence of the terrain on the data of aeromagnetic survey. The generalised model characteristics, obtained for the whole data set, are in agreement with the results from the experimental profiles. It proved that the preliminary estimations were correct and the proposed sequence of analysis was valid. The revealed interrelations between the components of the magnetic field and the relief made it possible to suppress the influence of the trap cover on the anomalous magnetic field and to distinguish weakly manifested geological bodies and structures.
\end{abstract}

Keywords: empirical mode decomposition method, group method of data handling, aeromagnetic survey, assessment of terrain influence.

Funding: The study was supported by grants of the Russian Foundation for Basic Research no. 19-05-00654 A and no. 18-05-70094 "Arctic Resources".

For citation: Muravina O. M., Dolgal A. S., Ponomarenko I.A. Application of statistical methods to take into account the influence of the terrain when conducting aeromagnetic surveys. Vestnik Voronezhskogo gosudarstvennogo universiteta. Seriya: Geologiya = Proceedings of Voronezh State University. Series: Geology. 2020. No. 2. pp. 69-75. DOI: https://doi.org/10.17308/geology.2020.2/2860

Acknowledgments: The authors are grateful to prof. V. N. Glaznev for constructive comments and useful discussion of research materials.

Conflict of interests: The authors declare the absence of obvious and potential conflicts of interest related to the publication of this article

๑ Olga M. Muravina, e-mail: muravina@geol.vsu.ru 


\section{REFERENCES}

1. Krivoluczkaya N. A. E`volyuciya trappovogo magmatizma i Pt-Cu-Ni rudoobrazovaniya v Noril'skom rajone. [The evolution of trap magmatism and $\mathrm{Pt}-\mathrm{Cu}-\mathrm{Ni}$ ore formation in the Norilsk region] Tovarishhestvo nauchny`x izdanij KMK. 2014. 305 p. (in Russ).

2. Rempel' G. G. Aktual'ny'e voprosy' metodiki vvedeniya popravok, svyazanny`x s rel`efom mestnosti danny`e gravirazvedki i magnitorazvedki [Actual issues of the methodology for introducing amendments related to the terrain, data of gravity and magnetic exploration]. Trudy Akademii nauk SSSR. Serii. Fizika Zemli = Proceedings of the USSR Academy of Sciences. Series. Earth Physics. 1980, no 12 , pp. 75-89. (in Russ.)

3. Dolgal A. S., Lazarev F. D., Kanunnikov V. A., Kirplyuk P. V. Uchet vliyaniya rel'efa mestnosti na rezul taty' krupnomasshtabnoj ae romagnitnoj s"emki [Consideration of the effect of the terrain on the results of large-scale aeromagnetic surveys]. Voprosy 'teorii $i$ praktiki geologicheskoj interpretacii gravitacionny`x, magnitny`x $i$ e`lektricheskix polej: materialy`42-j sessii Mezhdunar. nauch. seminara im. D.G. Uspenskogo. [Practical and Theoretical Aspects of Geological Interpretation of Gravitational, Magnetic and Electric Fields: proceedings of the 42th Uspensky International Geophysical Seminar], Perm, January 26-30, 2015, pp. 73-75 (in Russ.)

4. Dolgal A. S. Magnitorazvedka: komp 'yuterny'e texnologii ucheta vliyaniya rel'efa mestnosti [Magnetic exploration: computer technology for taking into account the influence of the terrain] Perm, Publ PSSRU. 2014. 92 p. (in Russ.)

5. Dolgal A. S. Allowance for the Earth's Surface Topography in Processing the Magnetic Field Measurements. In: Nurgaliev D., Khairullina N. (eds) Practical and Theoretical Aspects of Geological Interpretation of Gravitational, Magnetic and Electric Fields. Springer Proceedings in Earth and Environmental Sciences. Springer, Cham. 2019, pp. 3-12. DOI https://doi.org/10.1007/978-3-319-97670-9_12. 6. Dolgal A. S., Chervonyj N. P. Uchet vliyaniya rel'efa zemnoj poverxnosti pri ae'romagnitny`x izmereniyax [Taking into account the influence of the relief of the earth's surface during aeromagnetic measurements]. Geoinformatics. 2008, no. 2, pp. 58-66. (in Russ.) 7. Dolgal A. S., Muravina O. M., Hristenko L. A. The reduction of the magnetic field within development areas of the plateaubasalts. Geoinformatics. 2017. 15-17 May 2017, Kiev, Ukraine. 11143 ENG. Electronic resources (CD).

8. Huang N .E., Shen Z.,. Long S. R, Wu M. C., Shih H. Y., Zheng Q., Yen N. C., Tung C. C., and Liu H.H. The empirical mode decomposition and the Hilbert spectrum for nonlinear and non-stationary time series analysis // Proc. R. Soc. Lond. A. 1998. V. 454. P. 903-995.

9. Dolgal A. S., Hristenko L. A. Primenenie e'mpiricheskoj modovoj dekompozicii pri obrabotke geofizicheskix danny`x [The use of empirical mode decomposition in the processing of geophysical data]. Bulletin of the Tomsk Polytechnic University. Geo Asset Engineering, 2017, vol. 328, no. 1, pp. 100-108. (in Russ.)

10. Ivahnenko A. G. Induktivnyj metod samoorganizacii modelej slozhnyh system [Inductive method of self-organization of models of complex systems] Kiev, Naukova Dumka publ., 1982. 296 p. (in Russ.) 11. Muravina O. M. Metod gruppovogo ucheta argumentov pri analize geofizicheskih dannyh [The method of group accounting of ar- guments in the analysis of geophysical data]. Geophysics, 2012, no. 6, pp. 10 - 20. (in Russ.)

12. Muravina, O. M. Davudova, I. A. Ponomarenko Possibility of identification of modeling in complex analysis geological and geophysical data. Practical and Theoretical Aspects of Geological Interpretation of Gravitational, Magnetic and Electric Fields: proceedings of the 45th Uspensky International Geophysical Seminar. Cep. "Springer Proceedings in Earth and Environmental Sciences". 2019. pp. 157-162.

13. Muravina O. M., Ponomarenko I. A. Programmnaya realizaciya metoda gruppo-vogo ucheta argumentov pri identifikacionnom modelirovanii geologo-geofizicheskih dannyh [Software implementation of the method of group accounting of arguments in identification modeling of geological and geophysical data]. Vestnik Voronezhskogo gosudarstvennogo universiteta. Ser. Geologiya $=$ Proceedings of Voronezh State University. Series: Geology. 2016, no. 2 pp. 107-110. (in Russ.) 14. Muravina O. M., Auzin A. A., Glaznev V. N., Ponomarenko I. A. $\mathrm{O}$ vozmozhnosti izucheniya kollektorskix svojstv porod metodom identifikacionnogo modelirovaniya [On the possibility of studying the reservoir properties of rocks by identification modeling]. Karotazhnik, 2018, no. 8, pp.71-80. (in Russ.)

15. Muravina O. M Identifikacionnyj analiz petrofizicheskih harakteristik porod osadochnogo chekhla Voronezhskoj anteklizy. Vestnik KRAUNC. Ser. Nauki o Zemle. 2013, vol. 22, no. 2, pp. 20 25. (in Russ.).

16. Muravina O. M. Vozmozhnosti metoda gruppovogo ucheta argumentov pri statisticheskoj obrabotke petrofizicheskih dannyh [Possibilities of the method of group accounting of arguments in the statistical processing of petrophysical data]. Vestnik Voronezhskogo gosudarstvennogo universiteta. Ser. Geologiya $=$ Proceedings of Voronezh State University. Series: Geology, 2009, no. 2, pp. 150-110. (in Russ.) 17. Muravina O. M, Chernyshova M. N., Zhavoronkin V. I. Identifikacionny $\mathrm{j}$ analiz ul tramafit-mafitovy 'x intruzij mamonskogo kompleksa Voronezhskogo kristallicheskogo massiva [Identification analysis of ultramafic-mafic intrusions in the mamonsky complex of the Voronezh crystalline massif]. Vestnik KRAUNC. Ser. Nauki o Zemle, 2019, vol. 43, no. 3, pp. 89-98, DOI: $\quad$ 10.31431/18165524- 019-3-43-89-98 (in Russ.)

18. Muravina O. M., Dolgal A. S., Auzin A. A., Ponomarenko I. A., Gruzdev V. N. Sfery' primeneniya sovremenny'x statisticheskix metodov obrabotki geofizicheskoj informacii [Areas of application of modern statistical methods for processing geophysical information]. Vestnik Voronezhskogo gosudarstvennogo universiteta. Ser. Geologiya $=$ Proceedings of Voronezh State University. Series: Geology. 2019, no. 4 pp. 79-84. (in Russ.)

19. Dolgal A. S., Muravina O. M., Krivoluczkaya N. A. Uchet vliyaniya rel'efa zemnoj poverxnosti pri reducirovanii ae'romagnitny`x izmerenij $\mathrm{v}$ predelax bol'shix magmaticheskix provincij [Taking into account the influence of the relief of the earth's surface during the reduction of aeromagnetic measurements within large magmatic provinces]. Glubinnoe stroenie, geodinamika, teplovoe pole Zemli: materialy`desyaty`x nauchny`x chtenij pamyati Yu.P. Bulashevicha [Deep structure, geodynamics, thermal field of the Earth: materials of tenth scientific readings in memory of Yu.P. Bulashevich]. Yekaterinburg, 2019. pp. 114-118.
Муравина Ольга Михайловна - д. тех. н., профессор, Воронежский государственный университет, Воронеж, РФ; Е-mail: muravina@geol.vsu.ru; ORCID http://orcid.org/0000-0003-4772-0825

Долгаль Александр Сергеевич - д. физ.-мат. н., главный научный сотрудник, Горный институт УрО РАН, Пермь, РФ; Е-mail: dolgal@mi-perm.ru; ORCID http://orcid.org/0000-0003-0099-3471

Пономаренко Иван Александрович - аспирант кафедры геофизики, Воронежский государственный университет, Воронеж, PФ; E-mail: kochuma@yandex.ru; ORCID http://orcid.org/00000002-2588-3917

Авторы прочитали и одобрили окончательный вариант рукописи.
Olga M. Muravina - Dr. habil. in Techn., Professor, Voronezh State University, Voronezh, Russian Federation; E-mail: muravina@geol.vsu.ru; ORCID http://orcid.org/0000-0003-4772-0825

Alexsandr S. Dolgal - Dr.habil. in Phys.- Math., Chief research fellow, Mining Institute, Ural Branch of Russian Academy of Sciences, Perm, Russian Federation; dolgal@mi-perm.ru; ORCID http://orcid.org/0000-0003-0099-3471

Ivan A. Ponomarenko - postgraduate student, Voronezh State University, Voronezh, Russian Federation; E-mail: kochuma@yandex.ru; ORCID http://orcid.org/0000-0002-2588-3917

All authors have read and approved the final manuscript. 